\title{
Meta-analysis of the effects of dexmedetomidine vs esmolol for used controlled hypotension during surgery
}

Jiuyi Li

The First People's Hospital of Chen Zhou

\section{Lingkun Huang}

The First People's Hospital of Chenzhou

\section{Zhiming Zhang}

The First People's Hospital of Chenzhou

\section{Bin Zeng}

The First People's Hospital of Chenzhou

\section{Huiyin Deng ( $\nabla$ huiyind@csu.edu.cn )}

the frist people's hospital of chen zhou https://orcid.org/0000-0001-5405-9719

\section{Research article}

Keywords: Dexmedetomidine, Esmolol, Controlled hypotension, Meta-analysis

Posted Date: June 16th, 2020

DOl: https://doi.org/10.21203/rs.3.rs-29871/v1

License: (9) This work is licensed under a Creative Commons Attribution 4.0 International License. Read Full License 


\section{Abstract}

Background: At present ,the anesthetist usally use Controlled hypotension tecnical reduced blood loss and improved surgical field. Dexmedetomidine and esmolol have been proved effective for controlled hypotension. This meta-analysis aimed to evaluate the effects of dexmedetomidine versus esmolol on the controlled hypotension during surgery.

Methods: All articles were retrieved from PubMed, Embase, Cochrane Library up to January 2020. JL and $\mathrm{HD}$ independently screened, extracted and evaluated the literature that meet the inclusion and exclusion criteria, and used RevMan 5.3 for statistic analysis.

Results: Eight randomised trials, including 418 patients were selected in this meta-anaysis. And it's results showed:1) The dexmedetomidine group was more favorable in reducing blood loss (MD 49.48 with 95\%

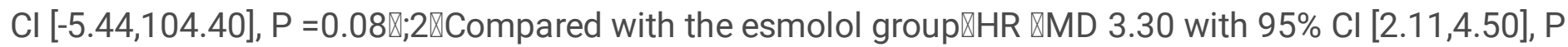
$<0.00001$, requiring of fentany(SMD 5.96 with $95 \% \mathrm{Cl}[3.43,8.48]$, $\mathrm{P}<0.00001$ \were considerably lower in the dexmedetomidine group $\llbracket$ while emergence time $\triangle \mathrm{MD}-6.69$ with $95 \% \mathrm{Cl}[-9.61,-3.76], \mathrm{P}<0.0001$ )was

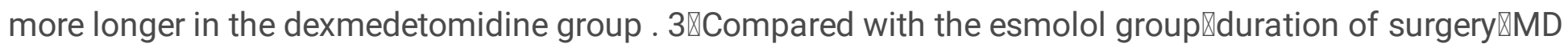

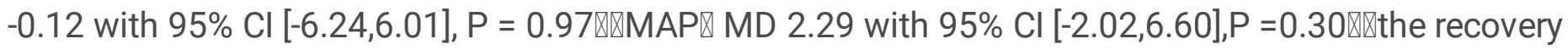
period $\triangle \mathrm{MD}-0.52$ with $95 \% \mathrm{Cl}[-3.07,2.04], \mathrm{P}=0.69 \llbracket$ were not statistically significant differences in the dexmedetomidine group.

Conclusion: Two drugs were effective for controlled hypotension, but compared with esmolol, dexmedetomidine is more better in reducing blood loss, and less requiring of fentanyl.But attention should be paid to the long emegence time and bradycardia.

\section{Background}

Controlled hypotension is the effectives method to reduce intraoperative bleeding.Nevertheless, both procedure and patients pathology can increase arterial blood pressure that give rise to excessive blood loss and adverse outcomes[1]. Intra-operative bleeding is associated with poor surgical field visibility, and increased surgical time for various surgery .Many drugs have been developed to limit blood loss during surgery. But these interventions are reported some adverse effects. Therefore, we remains to determine an ideal intervention to provide controlled hypotension.

Dexmedetomidine (a potent and highly selective 2 - adrenergic agonist) has many effecs: sedation, analgesia ,anesthesia, and sympatholytic properties. Alpha-2 adrenergic agonists can reduces sympathetic tone that results in a lower HR, blood pressure, and hemodynamic response. That drop of blood pressure are caused by the inhibited outflow of central sympathetic or the decreased norepinephrine release of presynaptic[2]. Esmolol ( a short-acting b-blocker) can effectively lower heart rate and blood pressure, resulting in controlled perioperative stress response.The fall of blood pressure are because the reduced cardiac output due to negative chronotropic or the ionotropic effects of $\beta$ - 
adrenergic was blocked. Esmolol can win a stable controlled hypotension , and beneficial for surgery and blood conservation[3].

Although many studies have confirmed that the above two drugs have significant clinical effects on intraoperative controlled hypotension[4,5], no systematic study has been founded to compare the efficacy of the two drugs. Therefore, this literature systematically reviews the available evidence and compares the two drugs in the control of hypotension.

\section{Methods}

\section{Search strategy}

Two reviewers (JL, HD) independently searched and retrieved relevant studies from PubMed, EMBASE, and the Cochrane Library up to January, 2020. No language or publication status restrictions were applied,and the study was limited to human. Noteexpress 3.0 was used to merge and remove duplicate documents.

\section{Inclusion and exclusion criteria}

Patients.Adult patients who have received general anesthesia and used dexmedetomidine or esmolol as hypotensive drug were required in this study.

Experiment and control. Trial groups: dexmedetomidine;control groups:esmolol

Outcome indicators. Anticipated indicators included blood loss,Mean arterial pressure(MAP), Heart rate(HR), duration of surgery, requiring of fentanyl, and the recovery period (The time required to reach Aldrete score >9)[6] and emergence time[7](the interval between discontinuation of the anesthetics to response of eye opening to the verbal command).

Studies. The literature eligible for inclusion in this study should be randomized controlled trial. animal studies, reviews, case reports, or protocols were eliminated.

\section{Data Extraction and Quality Assessment}

J. Li and H. Deng separate review these studys for data that meet the inclusion and exclusion criteria. After scanned, two reviewers cross-checked the data.and resolved disagreements through discussion or the third author.The Cochrane Handbook for Systematic Reviews of Interventions was used to scan each study for methodological quality. Evaluation contents included: random sequence generation, allocation concealment, blinding of participants and personnel, blinding of outcome assessment, incomplete outcome data, selective reporting and other bias. These categories include : low risk of bias, unclear of bias and high risk of bias.

\section{Statistical Analysis}


We choose RevMan 5.3 for this meta-analysis. The mean difference (MD) or standard error of the mean (SEM) and 95\% confidence interval (Cl) were described for the continuous data, and the risk ratio (RR) , $95 \%$ confidence interval $(\mathrm{Cl})$ for the numerical data. Follow the Cochrane guidelines, $\mathrm{I}^{2}$ test was used for quantitative heterogeneity, and $\mathrm{I}^{2} \geq 50 \%$ means significant heterogeneity. When the results had no significant heterogeneity, the fixed effect model was applied for this system review.Otherwise the random effect model was used. And $p<0.05$ was specified as significance level.

\section{Result}

The flowchart (Fig. 1) show the process of literature search and selection. A total of 115 articles were retrieved from three databases After excluded duplicating, The remaining 75 articles were identified for further assessment. In the end, eight RCTs were included[8-15] .

\section{Study Characteristics}

Table 1 displayed the Characteristics of eight RCTS

\section{Quality Assessment}

Figures 2 and 3 shown risk of bias of all the included study. As shown in the figure,the included RCTs showed good quality in risk of bias.

\section{Meta-Analysis Results}

\section{Blooding loss}

A total of there RCTs were included in the study, involving 110 patients, which compared intraoperative blood loss of dexmedetomidine and esmolol. The random effects model was used because of the high heterogeneity $\left(I^{2}=92 \%\right)$. The results indicated that there was no significant difference between the two group in reducing intraoperative blood loss (MD 49.48 with $95 \% \mathrm{Cl}[-5.44,104.40], \mathrm{P}=0.08$ ). The result is shown in Fig. 4

\section{HR}

A total of four RCTs were included in the study, involving 230 patients, which compared HR of dexmedetomidine and esmolol. The fixed effects model was applied because of significant heterogeneity $(12=34 \%)$. The results indicated that there was significant difference between the two group in HR. (MD 3.30 with $95 \% \mathrm{Cl}[2.11,4.50], \mathrm{P}<0.00001,12=34 \%)$. The result is shown in Fig. 5

\section{MAP}

A total of four RCTs were included in the study, involving 230 patients, which compared HR of dexmedetomidine and esmolol. The randomed effects model was applied because of higher 
heterogeneity $(12=95 \%)$. The results showed that there was no significant difference between the two group in MAP. (MD 2.29 with 95\% $\mathrm{Cl}[-2.02,6.60], \mathrm{I} 2=95 \%, \mathrm{P}=0.30)$. The result is shown in Fig. 6 .

\section{Duration of surgery}

All seven RCTs involving 338 patients was included. The heterogeneity test $\left(I^{2}=56 \%\right)$ indicates that heterogeneity was existence between the selected literatures in this study, and the randomed effect was applied for meta analysis. The results indicated that there was no significant difference in duration of surgery between the esmolol group and the dexmedetomidine group (MD -0.12 with $95 \% \mathrm{Cl}[-6.24,6.01], \mathrm{P}$ $=0.97)$. The result is shown in Fig. 7

\section{Requiring of fentanyl}

All five RCTS involving 278 patients were included. The heterogeneity test $\left(1^{2}=96 \%\right)$ indicates that heterogeneity was existence between the selected literatures in this study, and the randomed effect was selected for meta analysis. The results indicated that the dexmedetomidine group required less fentanyl than the esmolol group. (SMD 5.96 with $95 \% \mathrm{Cl}[3.43,8.48], \mathrm{P}<0.00001)$. The result is shown in Fig. 8

\section{Recovery period}

Three RCTs involving 148 patients were included. The heterogeneity test $\left(\mathrm{I}^{2}=87 \%\right)$ indicates that heterogeneity was existence between the selected literatures in this study, and the randomed effect was selected for meta analysis. The results of meta analysis indicated that the difference in the recovery period was not significant in two group. (MD -0.52 with $95 \% \mathrm{Cl}[-3.07,2.04], \mathrm{P}=0.69,12=87 \%$ ). The results are shown in Fig. 9.

\section{Emergence time}

Three RCTs involving 188 patients was included. The heterogeneity test $\left(\mathrm{I}^{2}=95 \%\right)$ indicates that heterogeneity was existence between the selected literatures in this study, and the randomed effect was selected for meta analysis.The results of meta analysis indicated that the interval between discontinuation of the anesthetics to response of eye opening to the verbal command in the esmolol group was more shorter than dexmedetomidine group.(MD -6.69 with 95\% $\mathrm{Cl}[-9.61,-3.76], \mathrm{P}<0.0001), 12$ =95\%). The results are shown in Fig. 10 .

\section{Discussion}

Deliberate hypotension is an important method to reduce intraoperative bleeding and maintain the perfusion of vital organs. The reduction of bleeding at the surgical site improves the quality of surgical field, reduces the number of operations and the occurrence of adverse reactions, and shorten the operation time[16, 17].This is the underlying concept for controlled hypotensive anesthesia[18],which is 
frequently used in neurosurgery, orthopaedic surgery, lumbar spine surgeries and middle ear and nose surgery $[19,20]$. A variety of hypotensive drugs included sodium nitroprusside,volatile anaesthetics, nitroglycerin, adenosine, sympathetic antagonists, hydralazine, trimethaphan,fenoldopam and a2 agonists have been applied to achieve controlled hypotension. These drugs have adverse effects [21] such as resistance to vasodilators, a longer post-sensory recovery period, cyanide toxicity, and the possibility of myocardial inhibition, which often limited the use in controlled hypotension.

The result of dexmedetomidine and esmolol in reducing blood loss of surgical site indicated the difference between two groups in reducing blood loss was not significant, but the outcomes indicated that dexmedetomidine group showed better reduction of bleeding. It may be resulted from two main reason: on the one hand,previous studies demonstrated that hemodynamic control and peripheral vasoconstriction of dexmedetomidine can reduce intraoperative bleeding and improve the operative field of vision;On the other hand,relevant studies[5] have shown that reduced the HR can significantly improve the field of vision in the operative area, rather than reduced the MAP to a dangerously low level and affected the perfusion of vital organs.In our study,compared with esmolol, HR was lower in the dexmedetomidine group,while MAP was not significant difference. The results were consistent with our study description of the advantages of dexmedetomidine in reducing blood loss.

The effect of the two drugs on the duration of surgery showed no significant difference between the two groups. In addition,this study has found dexmedetomidine can decrease the necessity of fentanyl administration during operation. This can be due to sedative, analgesic and anaesthetic sparing effects, and sympatholytic properties of dexmedetomidine[22].

In our study,we used two approach to assesse objectively the quality of postoperative recovery ,including recovery period and emergence time. The recovery period of patients were reported in all three studies,and the outcome from analysis indicated the recovery condition was not significant in two group.But form the result of emergence time, it was observed that patients receiveing dexmedetomidine needed longer time to response of eye opening to the verbal command.It could be explained that dexmedetomidine selectively binds to alpha-2adrenergic receptor with greater affinity ,and it need to take longer time to restoration when the drug diffuses out of its receptors.

Finally, the limitations of this study need to be taken into account.Although we conduct a comprehensive retrieval from the two aspects of retrieval strategy and retrieval database to the greatest extent, the sample size of this study was still insufficient.In addition, we did not conduct further subgroup analysis of surgical types to avoid heterogeneity in our meta-analysis. The next work is to further compare the adverse effects of two drugs in the perioperative period.To sum up, in order to ensure the reliability of the research conclusion, large sample size and more high-quality evidence is needed in the following work.

\section{Conclusion}

Both esmolol and dexmedetomidine was effective for controlled hypotension, but compared with esmolol, dexmedetomidine is more better in reducing blood loss, and less requiring of fentanyl.But 
attention should be paid to the long emegence time and bradycardia.

\section{Abbreviations}

MD: Mean difference; SEM: standard error of the mean ;RR: Relative risk; Cl: Confidence interval; MAP:Mean arterial pressure;HR: Heart rate; RCT: Randomized controlled trials;ASA:American Society of Anesthesiologists

\section{Declarations}

\section{Ethics approval and consent to participate}

Not applicable.

\section{Consent for publication}

Not applicable.

\section{Availability of data and materials}

All data generated or analyzed during this study are included in this published article.

\section{Competing interests}

The authors declare that they have no competing interests.

\section{Funding}

Not applicable.

\section{Authors' contributions}

$\mathrm{JL}$ and HD participated in the design, collected the data, performed statistical analyses, and drafted the manuscript. ZZ acted as critical revision of manuscript. All authors read and approved the final manuscript.

\section{Acknowledgements}

Not applicable.

\section{Authors' information}

${ }^{1}$ Department of Anesthesiology, The First People's Hospital of Chenzhou, Chenzhou, Hunan 423000, China. 
${ }^{2}$ Department of Anesthesiology, The Third Xiangya Hospital of Central South university , Changsha, Hunan 410013, China.

\section{References}

1. Goodnough LT, et al. Transfusion medicine. First of two parts-blood transfusion. N Engl J Med. 1999;340(6):438-47.

2. Hall JE, et al. Sedative, amnestic, and analgesic properties of small-dose dexmedetomidine infusions. Anesth Analg. 2000;90(3):699-705.

3. Degoute CS, et al. Remifentanil and controlled hypotension; comparison with nitroprusside or esmolol during tympanoplasty. Can J Anaesth. 2001;48(1):20-7.

4. Boezaart AP, van der Merwe J, Coetzee A. Comparison of sodium nitroprusside- and esmolol-induced controlled hypotension for functional endoscopic sinus surgery. Can J Anaesth. 1995;42(5 Pt 1):373-6.

5. Durmus $\mathrm{M}$, et al. Effect of dexmedetomidine on bleeding during tympanoplasty or septorhinoplasty. Eur J Anaesthesiol. 2007;24(5):447-53.

6. Aldrete JA. The post-anesthesia recovery score revisited. J Clin Anesth. 1995;7(1):89-91.

7. Chung F. Are discharge criteria changing? J Clin Anesth. 1993;5(6 Suppl 1):64S-68S.

8. Ruku R, et al. Randomized Open-Labelled Comparative Evaluation of the Efficacy of Nitroglycerine, Esmolol and Dexmedetomidine in Producing Controlled Hypotension in Spine Surgeries. Anesth Essays Res. 2019;13(3):486-91.

9. Bajwa SJ, et al. Nitroglycerine, esmolol and dexmedetomidine for induced hypotension during functional endoscopic sinus surgery: A comparative evaluation. J Anaesthesiol Clin Pharmacol. 2016;32(2):192-7.

10. Nazir O, et al., Use of dexmedetomidine and Esmolol for hypotension in lumbar spine surgery. 2016. 21(3).

11. Das A, et al. Controlled hypotension in day care functional endoscopic sinus surgery: A comparison between esmolol and dexmedetomidine: A prospective, double-blind, and randomized study. Saudi Journal of Anaesthesia. 2016;10(3):276-82.

12. Maghawry KM, et al. Cerebral oxygen saturation monitoring during hypotensive anesthesia in shoulder arthroscopy: A comparative study between dexmedetomidine and esmolol. Egyptian Journal of Anaesthesia. 2015;31(1):43-52.

13. Erbesler ZA, et al. A Comparison of the Effects of Esmolol and Dexmedetomidine on the Clinical Course and Cost for Controlled Hypotensive Anaesthesia. Turk J Anaesthesiol Reanim. 2013;41(5):156-61.

14. Shams T, et al. Induced hypotension for functional endoscopic sinus surgery: A comparative study of dexmedetomidine versus esmolol. Saudi Journal of Anaesthesia. 2013;7(2):175-80. 
15. Kol IO, et al. Controlled hypotension with desflurane combined with esmolol or dexmedetomidine during tympanoplasty in adults: A double-blind, randomized, controlled trial. Curr Ther Res Clin Exp. 2009;70(3):197-208.

16. Baker AR, Baker AB. Anaesthesia for endoscopic sinus surgery. Acta Anaesthesiol Scand. 2010;54(7):795-803.

17. Newton MC, et al. Metabolic and hormonal responses to induced hypotension for middle ear surgery. Br J Anaesth. 1996;76(3):352-7.

18. Tobias JD. Controlled hypotension in children: a critical review of available agents. Paediatr Drugs. 2002;4(7):439-53.

19. Boezaart AP, van der Merwe J, Coetzee A. Comparison of sodium nitroprusside- and esmolol-induced controlled hypotension for functional endoscopic sinus surgery. Can J Anaesth. 1995;42(5 Pt 1):373-6.

20. Szpalski M, Gunzburg R, Sztern B. An overview of blood-sparing techniques used in spine surgery during the perioperative period. Eur Spine J. 2004;13 Suppl 1:S18-27.

21. Degoute CS. Controlled hypotension: a guide to drug choice. Drugs. 2007;67(7):1053-76.

22. Bloor BC, et al. Effects of intravenous dexmedetomidine in humans. II. Hemodynamic changes. Anesthesiology. 1992;77(6):1134-42.

\section{Tables}

Table1: Baseline characteristicsof included studies.

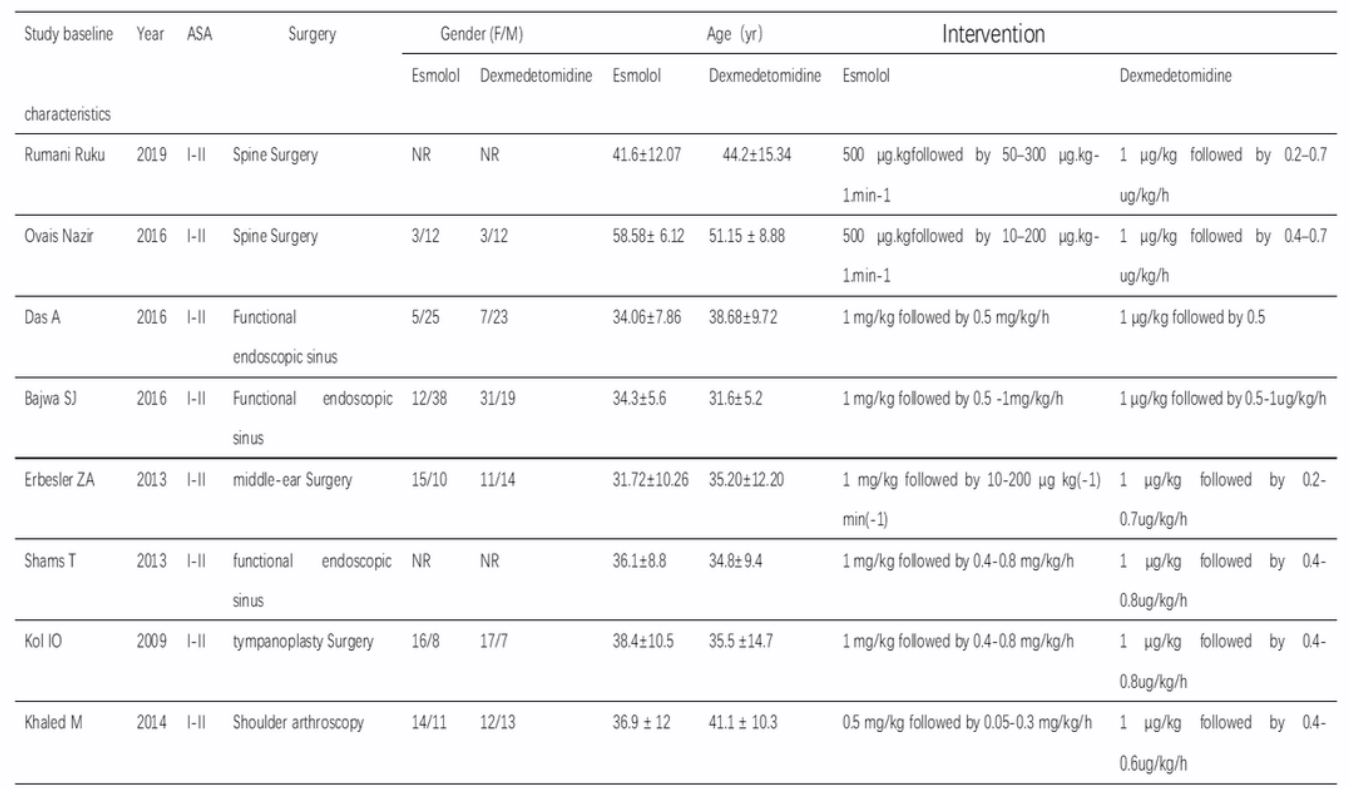

\section{Figures}


Random sequence generation (selection bias)

Allocation concealment (selection bias)

Blinding of participants and personnel (performance bias)

Blinding of outcome assessment (detection bias)

Incomplete outcome data (attrition bias)

Selective reporting (reporting bias)
Other bias

Selective reporting (reporting bias)
Other bias
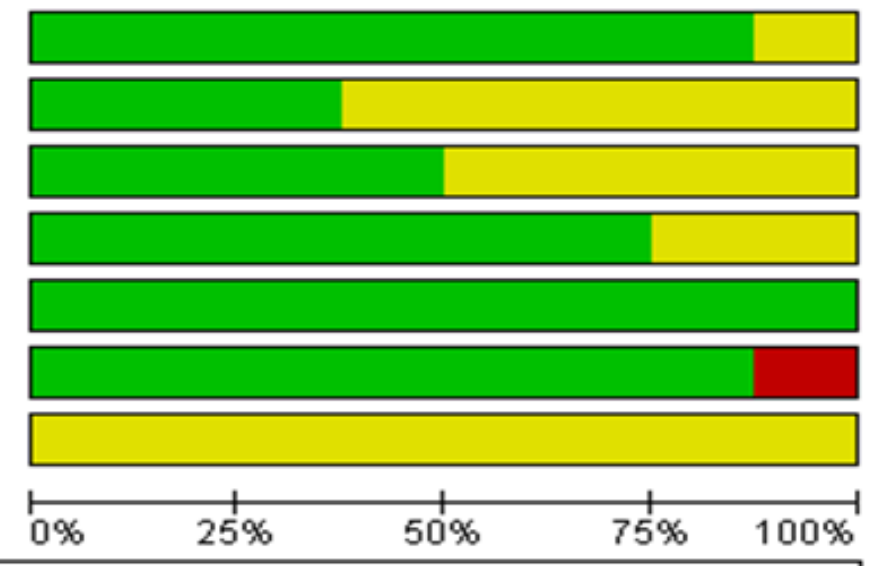

\section{Figure 1}

The risk of bias assessment of the included trials.

\begin{tabular}{|c|c|c|c|c|c|c|c|c|}
\hline 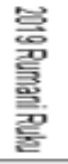 & 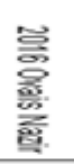 & 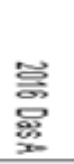 & 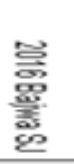 & $\begin{array}{l}\text { 总 } \\
\text { 兽 } \\
\text { 总 }\end{array}$ & 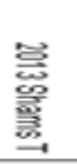 & 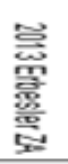 & $\begin{array}{l}\text { 总 } \\
\text { 至 }\end{array}$ & \\
\hline+ & + & + & + & + & + & 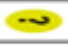 & $\rightarrow$ & \multirow{7}{*}{$\begin{array}{l}\text { Random sequence generation (selection bias) } \\
\text { Allocation concealment (selection bias) } \\
\text { Blinding or participants and personnel (perrormance bias) } \\
\text { Blinding or outcome assessment (detection bias) } \\
\text { incomplete outcome data (attrition bias) } \\
\text { selective reporting (reporting bias) } \\
\text { Other bias }\end{array}$} \\
\hline+ & $\sim$ & $\sim$ & 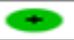 & $\approx$ & $\sim$ & $\sim$ & 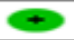 & \\
\hline$\rightarrow$ & + & + & + & + & $\rightarrow$ & $\rightarrow$ & $\sim$ & \\
\hline 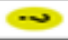 & + & + & + & + & + & $\sim$ & + & \\
\hline+ & $\leftarrow$ & 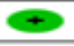 & 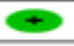 & 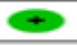 & $\leftarrow$ & + & 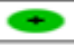 & \\
\hline+ & + & + & + & + & + & $\infty$ & + & \\
\hline 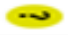 & $\approx$ & $\sim$ & $\sim$ & $\sim$ & $\approx$ & $\sim$ & $\sim$ & \\
\hline
\end{tabular}

\section{Figure 2}

The risk of bias summary of the included trials.

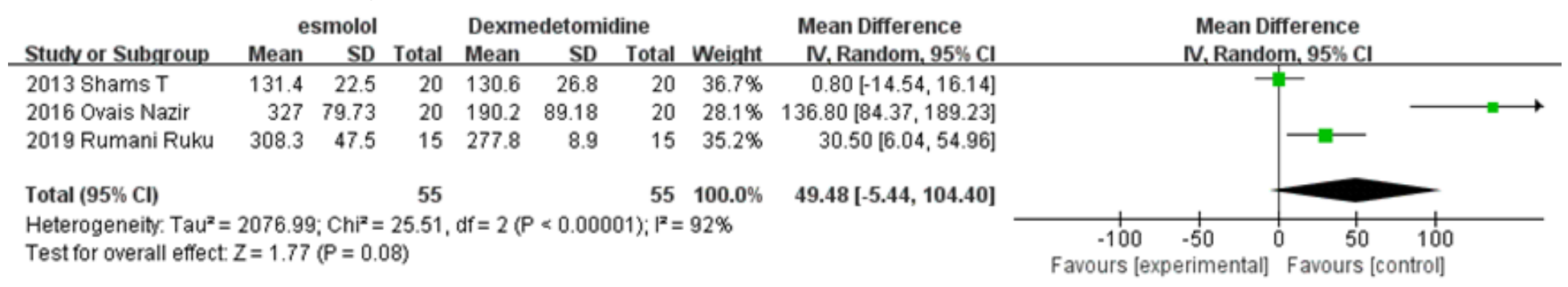

\section{Figure 3}

The effects of dexmedetomidine and esmolol in blooding loss 


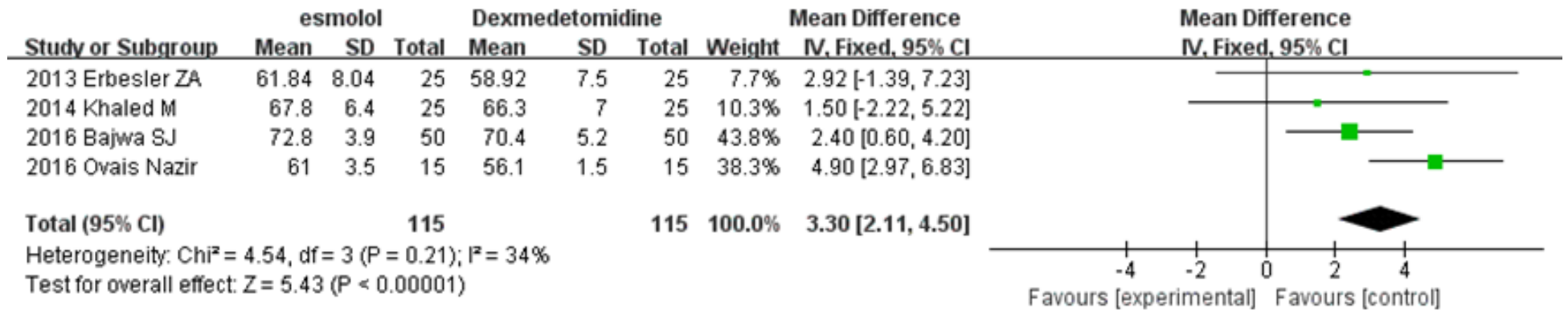

\section{Figure 4}

The effects of dexmedetomidine and esmolol in HR

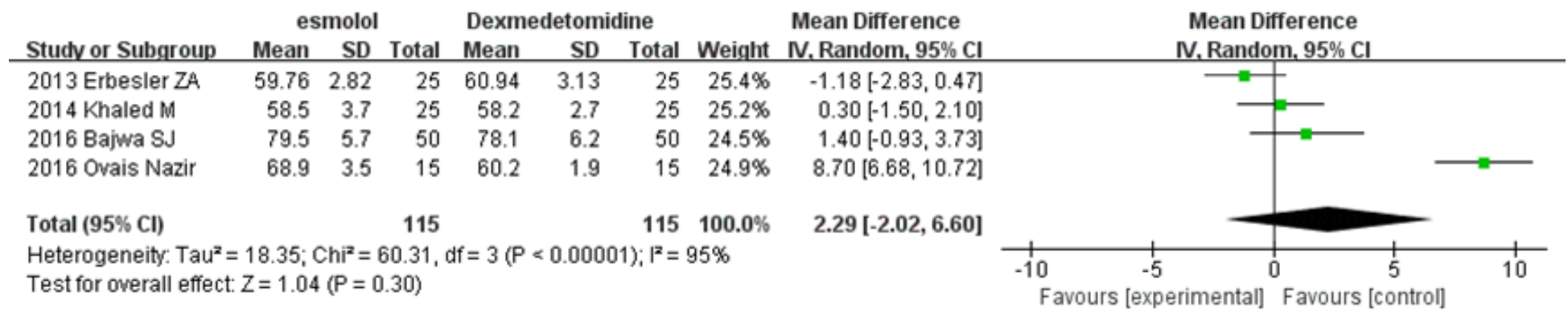

\section{Figure 5}

The effects of dexmedetomidine and esmolol in MAP

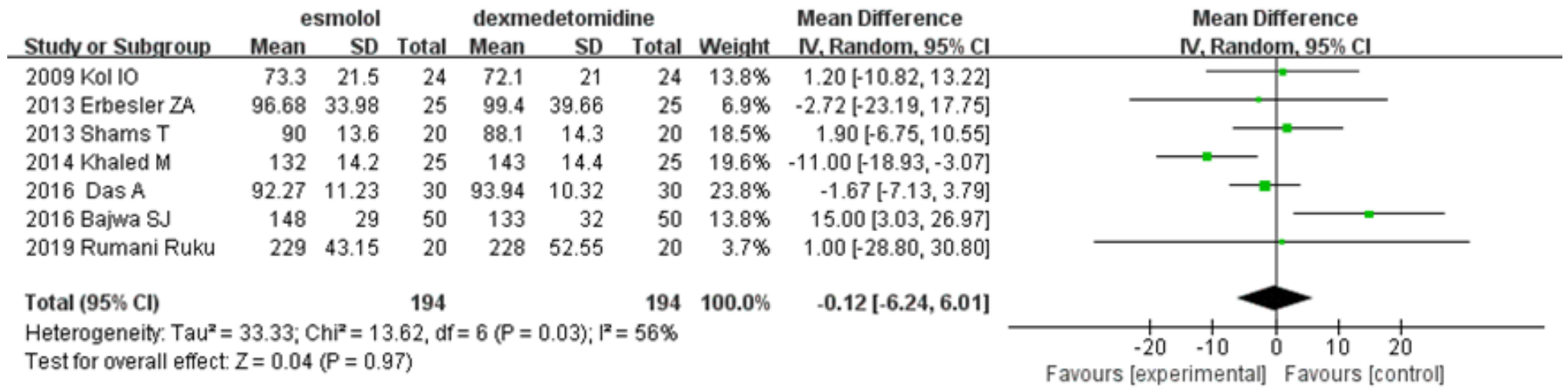

\section{Figure 6}

The effects of dexmedetomidine and esmolol in duration of surgery

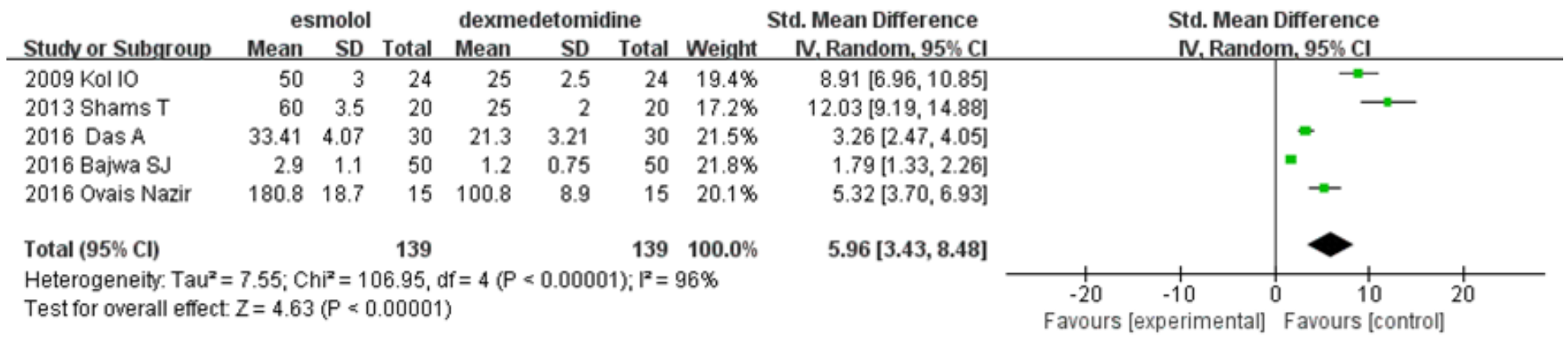


Figure 7

The effects of dexmedetomidine and esmolol in requiring of fentanyl

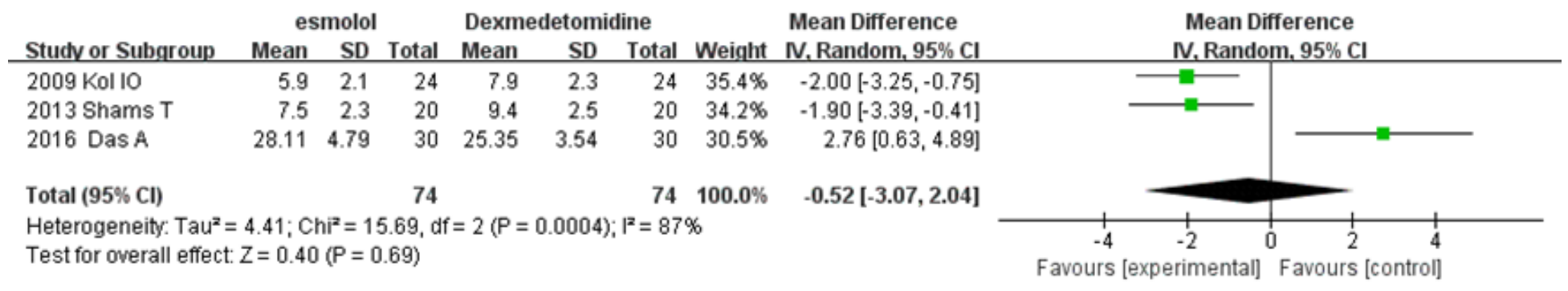

\section{Figure 8}

The effects of dexmedetomidine and esmolol in the recovery period

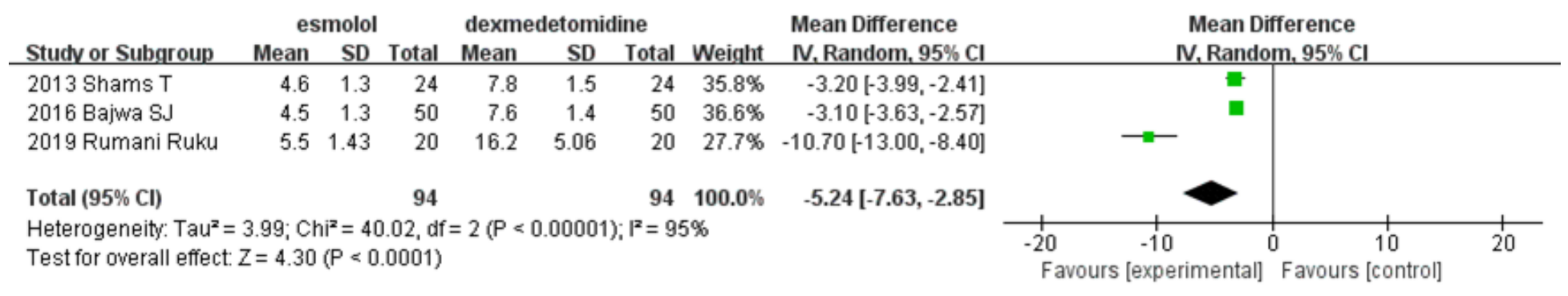

\section{Figure 9}

The effects of dexmedetomidine and esmolol in emergence time 
Records identified through database

searching ( $\mathrm{n}=115)$ : pubmed-25/embase -38

Cochrane library-52

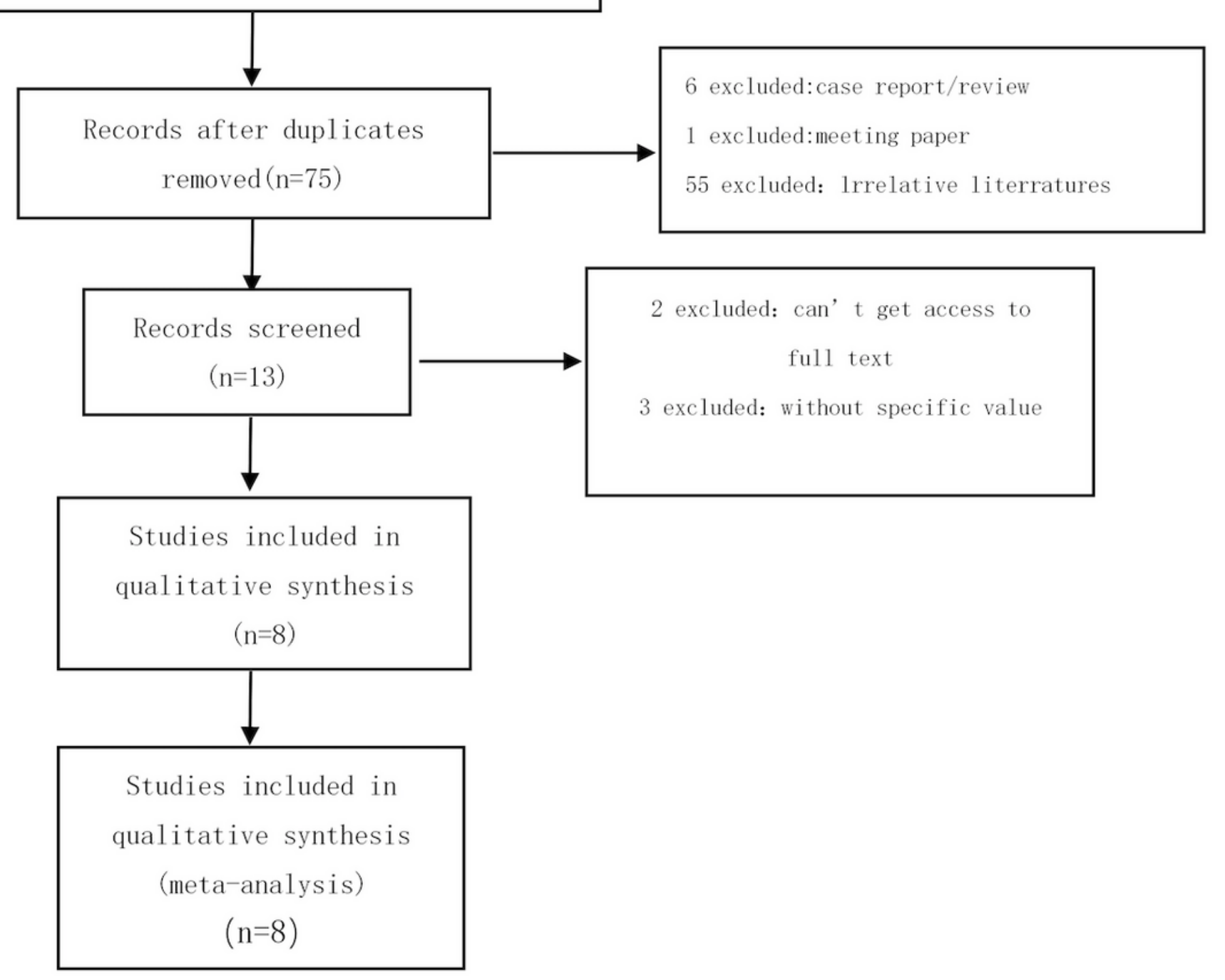

\section{Figure 10}

The flow chart of trial selection in the meta-analysis

\section{Supplementary Files}

This is a list of supplementary files associated with this preprint. Click to download.

- PRISMAchecklist.docx 